IRSTI 27.39 .15

$$
\text { https://doi.org/10.26577/JMMCS.2020.v105.i1.03 }
$$

${ }^{1}$ R. Oinarov, ${ }^{2}$ B.K. Omarbayeva, ${ }^{3}$ A.M. Temirkhanova

${ }^{1}$ Doctor of Physical and Mathematical Sciences, E-mail: o_ryskul@mail.ru

${ }^{2} \mathrm{PhD}$ student, E-mail: gaziza.omarbaeva@mail.ru

${ }^{3} \mathrm{PhD}$, E-mail: ainura-t@yandex.kz

L.N. Gumilyov Eurasian National University, Nur-Sultan, Kazakhstan

\title{
DISCRETE ITERATED HARDY-TYPE INEQUALITIES WITH THREE WEIGHTS
}

Discrete, continuous Hardy-type inequalities are of great importance and have numerous applications in harmonic analysis, in the theory of integral, differential and difference operators, in the theory of embeddings of function spaces and in other branches of mathematics. In recent years, weighted estimates for multidimensional Hardy-type operators have been intensively studied, which have an important application in the study of boundedness properties of operators from a Lebesgue weighted space to a local Morrey-type space, as well as in the study of bilinear operators in Lebesgue weighted spaces. The discrete case of Hardy type inequalities with three weights is an open problem. An inequality involving an iteration of the discrete Hardy operator is traditionally considered difficult to estimate because it contains three independent weight sequences and three parameters at their different ratios. In this paper we prove some new discrete iterated Hardy-type inequality involving three weights for the case $0<p \leq \min \{q, \theta\}$.

Key words: inequalities, Hardy-type operator, weight, sequences, discrete Lebesgue spaces.

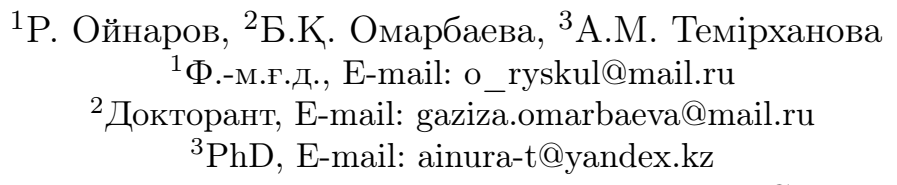

Л.Н. Гумилев атындағы Еуразия ұлттық университеті, Нұр-Сұлтан қ., Қазақстан Үш салмақты дискретті итерацияланған Харди типті теңсіздіктер

Дискретті, үзіліссіз Харди типті теңсіздіктері гармоникалық анализде, интегралдық, дифференциалдық және айырымдық операторлар теориясында, функционалдық кеңістіктердегі енгізу теориясында және басқада математиканың бөлімдерінде көптеген қолданыстары бар. Соңғы жылдары көп өлшемді Харди типті операторлардық салмақты бағалаулары қарқынды түрде зерттелуде, яғни олар Лебег салмақты кеңістігінен Морри типті локальді кеңістігіне шенелімділік қасиеттерін зерттеуде, сонымен қатар Лебег салмақты кеңістігіндегі бисызықты операторларды зерттеуде қолданылады. Үш салмақты Харди типті теңсіздігінің дискретті жағдайы ашық мәселе болып табылады. Әдетте, дискретті Харди операторының итерациясымен болатын теңсіздіктерді бағалау қиын деп саналады, өйткені оның құрамында үш тәуелсіз салмақты тізбек және әр түрлі қатынастағы үш параметр бар. Бұл мақалада біз кейбір жаңа үш салмақты дискретті итерацияланған Харди типті теңсіздікті $0<p \leq \min \{q, \theta\}$ жағдайы үшін дәлелдейміз.

Түйін сөздер: теңсіздік, Харди типті оператор, салмақ, тізбектер, дискретті Лебег кеңістігі.

$$
\begin{gathered}
{ }^{1} \text { Р. Ойнаров, }{ }^{2} \text { Б.К. Омарбаева, }{ }^{3} \text { A.M. Темирханова } \\
{ }^{1} \text { Д.ф.-м.н., E-mail: o_ryskul@mail.ru } \\
{ }^{2} \text { Докторант, E-mail: gaziza.omarbaeva@mail.ru } \\
{ }^{3} \text { PhD, E-mail: ainura-t@yandex.kz }
\end{gathered}
$$

Евразийский национальный университет имени Л.Н. Гумилева, г. Нур-Султан, Казахстан Дискретные итерационные неравенства типа Харди с тремя весами 
Дискретные, непрерывные неравенства типа Харди имеют большое значение и многочисленные приложения в гармоническом анализе, в теориях интегральных, дифференциальных и разностных операторов, в теории вложений функциональных пространств и в других разделах математики. В последние годы интенсивно исследуются весовые оценки для многомерных операторов типа Харди, которые имеют важное приложение в исследовании свойств ограниченности операторов из весового пространства Лебега в локальное пространство типа Морри, а также в исследовании билинейных операторов в весовых пространствах Лебега. Дискретный случай неравенств типа Харди с тремя весами является открытой проблемой. Неравенство, включающее итерацию дискретного оператора Харди, традиционно считается трудным для оценки, поскольку оно содержит три независимых весовых последовательностей и три параметра, при их различных соотношениях. В этой статье мы доказываем некоторое новое дискретное итерационное неравенство типа Харди с тремя весами для случая $0<p \leq \min \{q, \theta\}$.

Ключевые слова: неравенство, оператор типа Харди, вес, последовательности, дискретное пространство Лебега.

\section{Introduction}

Let $0<p, q, \theta<\infty$ and $\varphi=\left\{\varphi_{k}\right\}_{k=1}^{\infty}$ be a non-negative sequence, $u=\left\{u_{i}\right\}_{i=1}^{\infty}, \omega=\left\{\omega_{i}\right\}_{i=1}^{\infty}$ be positive sequences of real numbers, which will be referred to as weight sequences. We denote by $l_{p, u}$ the space of sequences $f=\left\{f_{j}\right\}_{j=1}^{\infty}$ of real numbers such that

$$
\|f\|_{p, u}=\left(\sum_{j=1}^{\infty}\left|u_{j} f_{j}\right|^{p}\right)^{\frac{1}{p}}<+\infty, \quad 1 \leq p<\infty .
$$

In this paper we characterize the following discrete Hardy-type inequalities:

$$
\left(\sum_{n=1}^{\infty} \omega_{n}^{\theta}\left(\sum_{k=n}^{\infty}\left|\varphi_{k} \sum_{i=k}^{\infty} f_{i}\right|^{q}\right)^{\frac{\theta}{q}}\right)^{\frac{1}{\theta}} \leq C\left(\sum_{j=1}^{\infty}\left|u_{j} f_{j}\right|^{p}\right)^{\frac{1}{p}}, \quad \forall f \in l_{p, u}
$$

for the following cases:

a) $1<p \leq \min \{q, \theta\}<\infty$,

b) $p \in(0,1]$ and $p \leq \min \{q, \theta\}<\infty$,

where $C$ is a positive constant independent of $f$.

Note that our result in case b) is especially interesting, since a continuous analogue of inequality (1) doesn't exist in this case.

\section{Literature review}

Since last century, one-dimensional discrete, continuous weighted Hardy inequalities have been investigated intensively in various functional spaces. The results of these works can be seen in the books of such authors, as for example B. Opic, A. Kufner, L. Maligranda, L.-E. Persson and N. Samko ([1]-[3]). In recent years, the general cases of the discrete, continuous weighted Hardy inequalities are investigated. For example the papers [4]-[15] have been devoted to the continuous analogue of discrete Hardy-type inequalities (1). An interest 
in this type of inequalities are caused by their applicability to spaces of the Morrey type ([16], [17]). Moreover, the characterizations of these inequalities can be applied to research weighted inequalities for Hardy's bilinear inequalities ([18]-[20]). However, the discrete Hardytype inequality (1) is study very little. For example, see the papers [21] and [22], where in particular, in [22] a criterion for the fulfilment of inequality (1) was obtained for the case $0<q<\theta<p<\infty, p>1$.

\section{Material and research methods}

The research methods are as follows: in this paper a method of partition of the sequence of elements of the Hardy operator on the part in each point is developed, which allows us to effectively estimate the sum on the parts. Note that such "blocking technic" was developed in [4]. During the estimate, various classical inequalities are used, such as Minkowski inequality, Holder inequality and the following elementary inequalities:

If $a_{i}>0, i=1,2, \ldots, k$, then

$$
\left(\sum_{m=1}^{k} a_{i}\right)^{\alpha} \leq \sum_{m=1}^{k} a_{i}^{\alpha}, \quad 0<\alpha \leq 1,
$$

and

$$
\left(\sum_{m=1}^{k} a_{i}\right)^{\alpha} \geq \sum_{m=1}^{k} a_{i}^{\alpha}, \quad \alpha \geq 1 .
$$

In the proofs of our main results we will need the following well-known version of the discrete Minkowski inequality:

Lemma. Let $\left\{a_{i, j}\right\}, i=1,2, \ldots, n \leq+\infty, j=1,2, \ldots, m$, be a positive matrix. Then the inequalities

$$
\left(\sum_{i=1}^{n}\left|\sum_{j=1}^{m} a_{i, j}\right|^{\sigma}\right)^{\frac{1}{\sigma}} \leq \sum_{j=1}^{m}\left(\sum_{i=1}^{n}\left|a_{i, j}\right|^{\sigma}\right)^{\frac{1}{\sigma}},
$$

and

$$
\left(\sum_{i=1}^{n}\left|\sum_{j=1}^{i} a_{i, j}\right|^{\sigma}\right)^{\frac{1}{\sigma}} \leq \sum_{j=1}^{n}\left(\sum_{i=j}^{n}\left|a_{i, j}\right|^{\sigma}\right)^{\frac{1}{\sigma}},
$$

hold, where $\sigma \geq 1$.

Convention: The symbol $M \ll K$ means that $M \leq c K$, where $c>0$ is a constant depending only on unessential parameters. If $M \ll K \ll M$, then we write $M \approx K$.

\section{Results and discussion}

\subsection{Main result}

Our main result reads as follows. 
Theorem. (i) If $1<p \leq \min \{q, \theta\}<\infty$, then the inequality (1) holds, if and only if $A_{1}<\infty$, where

$$
A_{1}:=\sup _{r \geq 1}\left(\sum_{n=1}^{r} \omega_{n}^{\theta}\left(\sum_{k=n}^{r} \varphi_{k}^{q}\right)^{\frac{\theta}{q}}\right)^{\frac{1}{\theta}}\left(\sum_{i=r}^{\infty} u_{i}^{-p^{\prime}}\right)^{\frac{1}{p^{\prime}}} .
$$

Moreover, $C \approx A_{1}$, where $C$ is the best constant in (1).

(ii) If $p \in(0,1]$ and $p \leq \min \{q, \theta\}<\infty$, then the inequality (1) holds, if and only if $A_{2}<\infty$, where

$$
A_{2}:=\sup _{r \geq 1}\left(\sum_{n=1}^{r} \omega_{n}^{\theta}\left(\sum_{s=n}^{r} \varphi_{s}^{q}\right)^{\frac{\theta}{q}}\right)^{\frac{1}{\theta}} \sup _{r \leq k} u_{k}^{-1} .
$$

Moreover, $C \approx A_{2}$, where $C$ is the best constant in (1).

Proof. Necessity: Suppose that the inequality (1) holds with best constant $C>0$.

(i) Let us show that $A_{1}<\infty$. Let $1 \leq r<N<\infty$ and take a test sequence $\widetilde{f}=\left\{\tilde{f}_{s}\right\}_{s=1}^{\infty}$ such that $\widetilde{f}_{s}=0$ for $1 \leq s<r$ and $s>N$ and $\widetilde{f}_{s}=u_{s}^{-p^{\prime}}$ for $r \leq s \leq N<\infty$.

Then

$$
\|\widetilde{f}\|_{l_{p, u}}=\left(\sum_{s=1}^{\infty}\left|\widetilde{f}_{s} \cdot u_{s}\right|^{p}\right)^{\frac{1}{p}}=\left(\sum_{s=r}^{N}\left|u_{s}^{-p^{\prime}} \cdot u_{s}\right|^{p}\right)^{\frac{1}{p}}=\left(\sum_{s=r}^{N} u_{s}^{-p^{\prime}}\right)^{\frac{1}{p}} .
$$

By substituting $\widetilde{f}$ in the left hand side of inequality (1), we can deduce that

$$
\begin{gathered}
I(\widetilde{f}):=\left(\sum_{n=1}^{\infty} \omega_{n}^{\theta}\left(\sum_{k=n}^{\infty}\left|\varphi_{k} \sum_{i=k}^{\infty} \widetilde{f}_{i}\right|^{q}\right)^{\frac{\theta}{q}}\right)^{\frac{1}{\theta}} \geq\left(\sum_{n=1}^{r} \omega_{n}^{\theta}\left(\sum_{k=n}^{N}\left|\varphi_{k} \sum_{i=k}^{N} \widetilde{f}_{i}\right|^{q}\right)^{\frac{\theta}{q}}\right)^{\frac{1}{\theta}} \geq \\
\geq\left(\sum_{n=1}^{r} \omega_{n}^{\theta}\left(\sum_{k=n}^{r}\left|\varphi_{k} \sum_{i=r}^{N} u_{i}^{-p^{\prime}}\right|^{q}\right)^{\frac{\theta}{q}}\right)^{\frac{1}{\theta}} \geq\left(\sum_{n=1}^{r} \omega_{n}^{\theta}\left(\sum_{k=n}^{r} \varphi_{k}^{q}\right)^{\frac{\theta}{q}}\right)^{\frac{1}{\theta}}\left(\sum_{i=r}^{N} u_{i}^{-p^{\prime}}\right) .
\end{gathered}
$$

From (1), (6) and (7), it follows that

$$
C \geq\left(\sum_{n=1}^{r} \omega_{n}^{\theta}\left(\sum_{k=n}^{r} \varphi_{k}^{q}\right)^{\frac{\theta}{q}}\right)^{\frac{1}{\theta}}\left(\sum_{i=r}^{N} u_{i}^{-p^{\prime}}\right)^{\frac{1}{p^{\prime}}}, \text { for all } 1 \leq r<N .
$$

Since $r \geq 1$ is arbitrary, passing to the limit as $N \rightarrow \infty$, we have that

$$
A_{1}=\sup _{r \geq 1}\left(\sum_{n=1}^{r} \omega_{n}^{\theta}\left(\sum_{k=n}^{r} \varphi_{k}^{q}\right)^{\frac{\theta}{q}}\right)^{\frac{1}{\theta}}\left(\sum_{i=r}^{\infty} u_{i}^{-p^{\prime}}\right)^{\frac{1}{p^{\prime}}} \leq C .
$$

(ii) Let us show that $A_{2}<\infty$. Now for $1<r \leq k<\infty$ we assume that $\widetilde{g}=\left\{\widetilde{g}_{s}\right\}_{s=1}^{\infty}$, where $\widetilde{g}_{s}=0$ for $s \neq k$ and $\widetilde{g}_{s}=u_{k}^{-1}$ for $s=k$, where $u_{k} \neq 0$. Then

$$
\|\widetilde{g}\|_{l_{p, u}}=u_{k}^{-1} \cdot u_{k}=1 .
$$

Substituting $\widetilde{g}$ in the left hand side of inequality (1), we find that

$$
I(\widetilde{g}):=\left(\sum_{n=1}^{\infty} \omega_{n}^{\theta}\left(\sum_{s=n}^{\infty}\left|\varphi_{s} \sum_{i=s}^{\infty} \widetilde{g}_{i}\right|^{q}\right)^{\frac{\theta}{q}}\right)^{\frac{1}{\theta}} \geq\left(\sum_{n=1}^{r} \omega_{n}^{\theta}\left(\sum_{s=n}^{r} \varphi_{s}^{q}\right)^{\frac{\theta}{q}}\right)^{\frac{1}{\theta}} u_{k}^{-1}, \quad \forall k \geq r,
$$




$$
I(\widetilde{g}) \geq\left(\sum_{n=1}^{r} \omega_{n}^{\theta}\left(\sum_{s=n}^{r} \varphi_{s}^{q}\right)^{\frac{\theta}{q}}\right)^{\frac{1}{\theta}} \sup _{r \leq k} u_{k}^{-1}, \quad \forall r \geq 1
$$

Therefore

$$
I(\widetilde{g}) \geq \sup _{r \geq 1}\left(\sum_{n=1}^{r} \omega_{n}^{\theta}\left(\sum_{s=n}^{r} \varphi_{s}^{q}\right)^{\frac{\theta}{q}}\right)^{\frac{1}{\theta}} \sup _{r \leq k} u_{k}^{-1}=A_{2} .
$$

From (1), (9) and (10), we have that

$$
A_{2}=\sup _{r \geq 1}\left(\sum_{n=1}^{r} \omega_{n}^{\theta}\left(\sum_{s=n}^{r} \varphi_{s}^{q}\right)^{\frac{\theta}{q}}\right)^{\frac{1}{\theta}} \sup _{r \leq k} u_{k}^{-1} \leq C .
$$

Sufficiency: Now, we prove that the inequality (1) holds. Let $0 \leq f \in l_{p, u}$ be such that

$$
\sum_{i=1}^{\infty} f_{i}<\infty
$$

Let

$$
k_{1}:=\inf \left\{k \in Z: \sum_{i=1}^{\infty} f_{i} \leq 2^{k}\right\}
$$

then

$$
2^{k_{1}-1} \leq \sum_{i=1}^{\infty} f_{i}<2^{k_{1}} .
$$

We consider the sequence $\left\{j_{k}\right\}$, where $j_{k}$ are defined by

$$
j_{k}:=\min \left\{j \geq 1: \sum_{i=j}^{\infty} f_{i} \leq 2^{k_{1}-k+1}\right\} .
$$

We note that

$$
j_{1}=\min \left\{j \geq 1: \sum_{i=j}^{\infty} f_{i} \leq 2^{k_{1}}\right\}=1 .
$$

For all $k \geq 1$ it yields that

$$
\sum_{i=j_{k}}^{\infty} f_{i} \leq 2^{k_{1}-k+1}<\sum_{i=j_{k}-1}^{\infty} f_{i}
$$

Therefore the set of natural numbers $\mathbb{N}$ can be written

$$
\mathbb{N}=\underset{k \geq 1}{\cup}\left[j_{k}, j_{k+1}-1\right]
$$

Moreover,

$$
2^{k_{1}-m+1}<\sum_{i=j_{m}-1}^{\infty} f_{i}=\sum_{i=j_{m}-1}^{j_{m+1}-1} f_{i}+\sum_{i=j_{m+1}}^{\infty} f_{i}<\sum_{i=j_{m}-1}^{j_{m+1}-1} f_{i}+2^{k_{1}-(m+1)+1}, \quad m \geq 2 .
$$


Hence,

$$
\begin{gathered}
2^{k_{1}-m}<\sum_{i=j_{m}-1}^{j_{m+1}-1} f_{i}, m \geq 2 . \\
2^{k_{1}-m}=2^{k_{1}-(m+1)+1}=2 \cdot 2^{k_{1}-(m+1)}<2 \sum_{i=j_{m+1}-1}^{j_{m+2}-1} f_{i}, \quad m \geq 2 .
\end{gathered}
$$

We have that

$$
2^{k_{1}-1+1}=2^{k_{1}}=4 \cdot 2^{k_{1}-2}<4 \sum_{i=j_{2}-1}^{j_{2+1}-1} f_{i}
$$

Then we obtain that

$$
2^{k_{1}-m+1}<4 \sum_{i=j_{m+1}-1}^{j_{m+2}-1} f_{i}, \quad m \geq 1
$$

Therefore, in view of (13),

$$
\begin{aligned}
I^{\theta}(f):=\sum_{n=1}^{\infty} \omega_{n}^{\theta}\left(\sum_{s=n}^{\infty}\left|\varphi_{s} \sum_{i=s}^{\infty} f_{i}\right|^{q}\right)^{\frac{\theta}{q}}=\sum_{k=1}^{\infty} \sum_{n=j_{k}}^{j_{k+1}-1} \omega_{n}^{\theta}\left(\sum_{s=n}^{\infty}\left|\varphi_{s} \sum_{i=s}^{\infty} f_{i}\right|^{q}\right)^{\frac{\theta}{q}} \leq \\
\leq \sum_{k=1}^{\infty} \sum_{n=j_{k}}^{j_{k+1}-1} \omega_{n}^{\theta}\left(\sum_{m=k}^{\infty} \sum_{s=\max \left(n, j_{m}\right)}^{j_{m+1}-1} \varphi_{s}^{q}\left(\sum_{i=j_{m}}^{\infty} f_{i}\right)^{q}\right)^{\frac{\theta}{q}} \leq \\
\leq \sum_{k=1}^{\infty} \sum_{n=j_{k}}^{j_{k+1}-1} \omega_{n}^{\theta}\left(\sum_{m=k}^{\infty} \sum_{s=\max \left(n, j_{m}\right)}^{j_{m+1}-1} \varphi_{s}^{q}\left(2^{k_{1}-m+1}\right)^{q}\right)^{\frac{\theta}{q}} .
\end{aligned}
$$

Hence, by applying (14) we have that

$$
I^{\theta}(f) \leq 4^{\theta} \sum_{k=1}^{\infty} \sum_{n=j_{k}}^{j_{k+1}-1} \omega_{n}^{\theta}\left(\sum_{m=k}^{\infty} \sum_{s=\max \left(n, j_{m}\right)}^{j_{m+1}-1} \varphi_{s}^{q}\left(\sum_{i=j_{m+1}-1}^{j_{m+2}-1} f_{i}\right)^{q}\right)^{\frac{\theta}{q}} .
$$

We must now consider the cases $\theta \leq q$ and $\theta>q$ separately.

4.2 The case $\theta \leq q$

(i) Let $1<p \leq \theta \leq q$. By applying (2) in (15), we find that

$$
I^{\theta}(f) \leq 4^{\theta} \sum_{k=1}^{\infty} \sum_{n=j_{k}}^{j_{k+1}-1} \omega_{n}^{\theta} \sum_{m=k}^{\infty}\left(\sum_{s=\max \left(n, j_{m}\right)}^{j_{m+1}-1} \varphi_{s}^{q}\right)^{\frac{\theta}{q}}\left(\sum_{i=j_{m+1}-1}^{j_{m+2}-1} f_{i}\right)^{\theta} .
$$


Now, by changing the orders of sums, we get that

$$
\begin{aligned}
& I^{\theta}(f) \leq 4^{\theta} \sum_{m=1}^{\infty}\left(\sum_{i=j_{m+1}-1}^{j_{m+2}-1} f_{i}\right)^{\theta} \sum_{k=1}^{m} \sum_{n=j_{k}}^{j_{k+1}-1} \omega_{n}^{\theta}\left(\sum_{s=\max \left(n, j_{m}\right)}^{j_{m+1}-1} \varphi_{s}^{q}\right)^{\frac{\theta}{q}} \leq \\
& \leq 4^{\theta} \sum_{m=1}^{\infty}\left(\sum_{i=j_{m+1}-1}^{j_{m+2}-1} f_{i}\right)^{\theta}\left(\sum_{n=j_{1}}^{j_{2}-1} \omega_{n}^{\theta}\left(\sum_{s=n}^{j_{m+1}-1} \varphi_{s}^{q}\right)^{\frac{\theta}{q}}+\sum_{k=2}^{m} \sum_{n=j_{k}}^{j_{k+1}-1} \omega_{n}^{\theta}\left(\sum_{s=j_{m}}^{j_{m+1}-1} \varphi_{s}^{q}\right)^{\frac{\theta}{q}}\right) \leq \\
& \leq 4^{\theta} \sum_{m=1}^{\infty}\left(\sum_{i=j_{m+1}-1}^{j_{m+2}-1} f_{i}\right)^{\theta}\left(\sum_{n=j_{1}}^{j_{2}-1} \omega_{n}^{\theta}\left(\sum_{s=n}^{j_{m+1}-1} \varphi_{s}^{q}\right)^{\frac{\theta}{q}}+\sum_{n=j_{2}}^{j_{m+1}-1} \omega_{n}^{\theta}\left(\sum_{s=j_{m}}^{j_{m+1}-1} \varphi_{s}^{q}\right)^{\frac{\theta}{q}}\right) \leq \\
& \leq 4^{\theta} \sum_{m=1}^{\infty}\left(\sum_{i=j_{m+1}-1}^{j_{m+2}-1} f_{i}\right)^{\theta}\left(\sum_{n=j_{1}}^{j_{2}-1} \omega_{n}^{\theta}\left(\sum_{s=n}^{j_{m+1}-1} \varphi_{s}^{q}\right)^{\frac{\theta}{q}}+\sum_{n=j_{2}}^{j_{m+1}-1} \omega_{n}^{\theta}\left(\sum_{s=n}^{j_{m+1}-1} \varphi_{s}^{q}\right)^{\frac{\theta}{q}}\right) \leq \\
& \leq 4^{\theta} \sum_{m=1}^{\infty}\left(\sum_{i=j_{m+1}-1}^{j_{m+2}-1} f_{i}\right)^{\theta} \sum_{n=j_{1}}^{j_{m+1}-1} \omega_{n}^{\theta}\left(\sum_{s=n}^{j_{m+1}-1} \varphi_{s}^{q}\right)^{\frac{\theta}{q}}
\end{aligned}
$$

so that

$$
I^{\theta}(f) \ll \sum_{m=1}^{\infty}\left(\sum_{i=j_{m+1}-1}^{j_{m+2}-1} f_{i}\right)^{\theta} \sum_{n=j_{1}}^{j_{m+1}-1} \omega_{n}^{\theta}\left(\sum_{s=n}^{j_{m+1}-1} \varphi_{s}^{q}\right)^{\frac{\theta}{q}} .
$$

Therefore, by using Holder's inequality and (3) in (16), we obtain that

$$
\begin{aligned}
& I^{\theta}(f) \leq \sum_{m=1}^{\infty}\left(\sum_{i=j_{m+1}-1}^{j_{m+2}-1}\left|f_{i} \cdot u_{i}\right|^{p}\right)^{\frac{\theta}{p}} \sum_{n=j_{1}}^{j_{m+1}-1} \omega_{n}^{\theta}\left(\sum_{s=n}^{j_{m+1}-1} \varphi_{s}^{q}\right)^{\frac{\theta}{q}}\left(\sum_{i=j_{m+1}-1}^{j_{m+2}-1} u_{i}^{-p^{\prime}}\right)^{\frac{\theta}{p^{\prime}}} \leq \\
& \left.\leq\left(\sum_{m=1}^{\infty} \sum_{i=j_{m+1}-1}^{j_{m+2}-1}\left|f_{i} \cdot u_{i}\right|^{p}\right)^{\frac{\theta}{p}}\left(\sup _{m \geq 1}\left(\sum_{n=j_{1}}^{j_{m+1}-1} \omega_{n}^{\theta}\left(\sum_{s=n}^{j_{m+1}-1} \varphi_{s}^{q}\right)^{\frac{\theta}{q}}\right)_{i=j_{m+1}-1}^{\frac{1}{\theta}} u_{i}^{-p^{\prime}}\right)^{\frac{1}{p^{\prime}}}\right)^{\theta} \leq \\
& \leq\left(\sum_{i=1}^{\infty}\left|f_{i} u_{i}\right|^{p}\right)^{\frac{\theta}{p}}\left(\sup _{r \geq 1}\left(\sum_{n=1}^{r} \omega_{n}^{\theta}\left(\sum_{s=n}^{r} \varphi_{s}^{q}\right)^{\frac{\theta}{q}}\right)^{\frac{1}{\theta}}\left(\sum_{i=r}^{\infty} u_{i}^{-p^{\prime}}\right)^{\frac{1}{p^{\prime}}}=\left(A_{1}\|f\|_{p, u}\right)^{\theta} .\right.
\end{aligned}
$$

Hence,

$$
I(f) \ll A_{1}\|f\|_{p, u}, \text { if } 1<p \leq \theta \leq q .
$$


(ii) Let $0<p \leq 1$. We start with the inequality (16):

$$
I^{\theta}(f) \ll \sum_{m=1}^{\infty}\left(\sum_{i=j_{m+1}-1}^{j_{m+2}-1} f_{i} \cdot u_{i} \cdot u_{i}^{-1}\right)^{p \cdot \frac{\theta}{p}} \sum_{n=1}^{j_{m+1}-1} \omega_{n}^{\theta}\left(\sum_{s=n}^{j_{m+1}-1} \varphi_{s}^{q}\right)^{\frac{\theta}{q}} .
$$

By applying (2) with $0<p \leq 1$, we obtain that

$$
\begin{aligned}
I^{\theta}(f) \leq & \sum_{m=1}^{\infty}\left(\sum_{i=j_{m+1}-1}^{j_{m+2}-1}\left|f_{i} \cdot u_{i}\right|^{p}\right)^{\frac{\theta}{p}}\left(\max _{j_{m+1}-1 \leq i \leq j_{m+2}-1} u_{i}^{-1}\right)^{\theta} \sum_{n=j_{1}}^{\theta j_{m+1}-1} \omega_{n}^{\theta}\left(\sum_{s=n}^{j_{m+1}-1} \varphi_{s}^{q}\right)^{\frac{\theta}{q}} \leq \\
& \leq \sum_{m=1}^{\infty}\left(\sum_{i=j_{m+1}-1}^{j_{m+2}-1}\left|f_{i} \cdot u_{i}\right|^{p}\right)^{\frac{\theta}{p}}\left(\sup _{j_{m+1}-1 \leq k} u_{k}^{-1}\right)^{\theta} \sum_{n=1}^{j_{m+1}-1} \omega_{n}^{\theta}\left(\sum_{s=n}^{j_{m+1}-1} \varphi_{s}^{q}\right)^{\frac{\theta}{q}} .
\end{aligned}
$$

By using (3), we get

$$
\begin{aligned}
I^{\theta}(f) & \leq\left(\sum_{m=1}^{\infty} \sum_{i=j_{m+1}-1}^{j_{m+2}-1}\left|f_{i} \cdot u_{i}\right|^{p}\right)^{\frac{\theta}{p}}\left[\sup _{m \geq 1}\left(\sum_{n=j_{1}}^{j_{m+1}-1} \omega_{n}^{\theta}\left(\sum_{s=n}^{j_{m+1}-1} \varphi_{s}^{q}\right)^{\frac{\theta}{q}} \sup _{j_{m+1}-1 \leq k} u_{k}^{-1}\right]^{\theta} \leq\right. \\
& \leq\left(\sum_{i=1}^{\infty}\left|f_{i} \cdot u_{i}\right|^{p}\right)^{\frac{\theta}{p}}\left[\sup _{r \geq 1}\left(\sum_{n=1}^{r} \omega_{n}^{\theta}\left(\sum_{s=n}^{r} \varphi_{s}^{q}\right)^{\frac{\theta}{q}}\right)^{\frac{1}{\theta}} \sup _{r \leq k} u_{k}^{-1}\right]^{\theta}=\left(A_{2}\|f\|_{p, u}\right)^{\theta},
\end{aligned}
$$

so that

$$
I(f) \ll A_{2}\|f\|_{p, u}, \text { if } p \leq \theta \leq q,
$$

where $p \in(0,1]$.

\subsection{The case $\theta>q$}

(i) Let $1<p \leq q<\theta$. We start with the inequality (15). First we raise both sides in (15) to the power $\frac{q}{\theta} \leq 1$, i.e.,

$$
I^{q}(f) \leq 4^{q}\left[\sum_{k=1}^{\infty} \sum_{n=j_{k}}^{j_{k+1}-1} \omega_{n}^{\theta}\left(\sum_{m=k}^{\infty} \sum_{s=\max \left(n, j_{m}\right)}^{j_{m+1}-1} \varphi_{s}^{q}\left(\sum_{i=j_{m+1}-1}^{j_{m+2}-1} f_{i}\right)^{q}\right]^{\frac{\theta}{q}} .\right.
$$

Next we apply (4) in the inner sum with $\sigma=\frac{\theta}{q}$ and obtain that

$$
I^{q}(f) \leq 4^{q}\left[\sum_{k=1}^{\infty}\left\{\sum_{m=k}^{\infty}\left(\sum_{n=j_{k}}^{j_{k+1}-1} \omega_{n}^{\theta}\left(\sum_{s=\max \left(n, j_{m}\right)}^{j_{m+1}-1} \varphi_{s}^{q}\right)^{\frac{\theta}{q}}\left(\sum_{i=j_{m+1}-1}^{j_{m+2}-1} f_{i}\right)^{\theta}\right\}^{\frac{q}{\theta}}\right]^{\frac{\theta}{q}},\right.
$$


Using (5), we get that

$$
\begin{aligned}
I^{q}(f) \leq 4^{q} \sum_{m=1}^{\infty}\left[\sum_{k=1}^{m} \sum_{n=j_{k}}^{j_{k+1}-1} \omega_{n}^{\theta}\left(\sum_{s=\max \left(n, j_{m}\right)}^{j_{m+1}-1} \varphi_{s}^{q}\right)^{\frac{\theta}{q}}\left(\sum_{i=j_{m+1}-1}^{j_{m+2}-1} f_{i}\right)^{\theta} \leq\right. \\
\leq 4^{q} \sum_{m=1}^{\infty}\left(\sum_{i=j_{m+1}-1}^{j_{m+2}-1} f_{i}\right)^{q}\left[\sum_{k=1}^{m} \sum_{n=j_{k}}^{j_{k+1}-1} \omega_{n}^{\theta}\left(\sum_{s=\max \left(n, j_{m}\right)}^{j_{m+1}-1} \varphi_{s}^{q}\right)^{\frac{\theta}{q}}\right]^{\frac{q}{\theta}} \leq \\
\leq 4^{q} \sum_{m=1}^{\infty}\left(\sum_{i=j_{m+1}-1}^{j_{m+2}-1} f_{i}\right)^{q}\left[\sum_{n=j_{1}}^{j_{m+1}-1} \omega_{n}^{\theta}\left(\sum_{s=n}^{j_{m+1}-1} \varphi_{s}^{q}\right)^{\frac{\theta}{q}}\right]^{\frac{q}{\theta}},
\end{aligned}
$$

so that

$$
I^{q}(f) \ll \sum_{m=1}^{\infty}\left(\sum_{i=j_{m+1}-1}^{j_{m+2}-1} f_{i}\right)^{q}\left[\sum_{n=j_{1}}^{j_{m+1}-1} \omega_{n}^{\theta}\left(\sum_{s=n}^{j_{m+1}-1} \varphi_{s}^{q}\right)^{\frac{\theta}{q}}\right]^{\frac{q}{\theta}} .
$$

Hence, by using Holder's inequality,

$$
I^{q}(f) \leq \sum_{m=1}^{\infty}\left(\sum_{i=j_{m+1}-1}^{j_{m+2}-1}\left|f_{i} \cdot u_{i}\right|^{p}\right)^{\frac{q}{p}}\left(\sum_{i=j_{m+1}-1}^{j_{m+2}-1} u_{i}^{-p^{\prime}}\right)^{\frac{q}{p^{\prime}}}\left[\sum_{n=j_{1}}^{j_{m+1}-1} \omega_{n}^{\theta}\left(\sum_{s=n}^{j_{m+1}-1} \varphi_{s}^{q}\right)^{\frac{\theta}{q}}\right]^{\frac{q}{\theta}} .
$$

Therefore, by applying (3) with $\alpha=\frac{q}{p}$, we obtain that

$$
\begin{aligned}
I^{q}(f) & \leq\left(\sum_{m=1}^{\infty} \sum_{i=j_{m+1}-1}^{j_{m+2}-1}\left|f_{i} \cdot u_{i}\right|^{p}\right)^{\frac{q}{p}}\left[\sup _{m \geq 1}\left(\sum_{n=j_{1}}^{j_{m+1}-1} \omega_{n}^{\theta}\left(\sum_{s=n}^{j_{m+1}-1} \varphi_{s}^{q}\right)^{\frac{\theta}{q}}\right)^{\frac{1}{\theta}}\left(\sum_{i=j_{m+1}-1}^{\infty} u_{i}^{-p^{\prime}}\right)^{\frac{1}{p^{\prime}}}\right]^{q} \leq \\
& \leq\left(\sum_{i=1}^{\infty}\left|f_{i} u_{i}\right|^{p}\right)^{\frac{q}{p}}\left(\sup _{r \geq 1}\left(\sum_{n=1}^{r} \omega_{n}^{\theta}\left(\sum_{s=n}^{r} \varphi_{s}^{q}\right)^{\frac{\theta}{q}}\right)^{\frac{1}{\theta}}\left(\sum_{i=r}^{\infty} u_{i}^{-p^{\prime}}\right)^{\frac{1}{p^{\prime}}}\right)^{q}=\left(A_{1}\|f\|_{p, u}\right)^{q},
\end{aligned}
$$

so that

$$
I(f) \ll A_{1}\|f\|_{p, u} \text {, when } 1<p \leq q<\theta .
$$

(ii) Let $0<p \leq 1$. We start with the inequality (19):

$$
I^{q}(f) \ll \sum_{m=1}^{\infty}\left(\sum_{i=j_{m+1}-1}^{j_{m+2}-1} f_{i} \cdot u_{i} \cdot u_{i}^{-1}\right)^{p \cdot \frac{q}{p}}\left(\sum_{n=1}^{j_{m+1}-1} \omega_{n}^{\theta}\left(\sum_{s=n}^{j_{m+1}-1} \varphi_{s}^{q}\right)^{\frac{\theta}{q}}\right)^{\frac{q}{\theta}} .
$$


By applying (2) with $0<p \leq 1$, we obtain that

$$
I^{q}(f) \leq \sum_{m=1}^{\infty}\left(\sum_{i=j_{m+1}-1}^{j_{m+2}-1}\left|f_{i} \cdot u_{i}\right|^{p}\right)^{\frac{q}{p}}\left[\sup _{j_{m+1}-1 \leq k} u_{k}^{-1}\left(\sum_{n=1}^{j_{m+1}-1} \omega_{n}^{\theta}\left(\sum_{s=n}^{j_{m+1}-1} \varphi_{s}^{q}\right)^{\frac{\theta}{q}}\right]^{\frac{1}{\theta}}\right]^{q}
$$

By using (3), we get that

$$
\begin{aligned}
I^{q}(f) & \leq\left(\sum_{m=1}^{\infty} \sum_{i=j_{m+1}-1}^{j_{m+2}-1}\left|f_{i} \cdot u_{i}\right|^{p}\right)^{\frac{q}{p}}\left[\sup _{m \geq 1}\left(\sum_{n=j_{1}}^{j_{m+1}-1} \omega_{n}^{\theta}\left(\sum_{s=n}^{j_{m+1}-1} \varphi_{s}^{q}\right)^{\frac{\theta}{q}} \sup _{j_{m+1}-1 \leq k} u_{k}^{-1}\right]^{q} \leq\right. \\
& \leq\left(\sum_{i=1}^{\infty}\left|f_{i} \cdot u_{i}\right|^{p}\right)^{\frac{q}{p}}\left[\sup _{r \geq 1}\left(\sum_{n=1}^{r} \omega_{n}^{\theta}\left(\sum_{s=n}^{r} \varphi_{s}^{q}\right)^{\frac{\theta}{q}}\right)^{\frac{1}{\theta}} \sup _{r \leq k} u_{k}^{-1}\right]^{q}=\left(A_{2}\|f\|_{p, u}\right)^{q},
\end{aligned}
$$

so that

$$
I(f) \ll A_{2}\|f\|_{p, u}, \text { if } p \leq q<\theta
$$

where $p \in(0,1]$.

The estimates (17), (20) and (18), (21) were obtained under the condition (12). Let $M=\left\{f \in l_{p, u}: \exists N=N(f)>0\right.$ and $\left.f_{i}=0, i \geq N\right\}$. Since $f$ from $M$ satisfy the condition (12) and the set $M$ is everywhere dense in $l_{p, u}$, then the estimates (17), (20) and (18), (21) are satisfied for all $f \in l_{p, u}$. Therefore from the inequalities (8), (17) and (20), we get $C \approx A_{1}$ and from the inequalities (8) and (18), (21), we get $C \approx A_{2}$, where $C$ is the best constant in (1). The proof of Theorem is complete.

\section{Conclusion}

In conclusion, we have established necessary and sufficient conditions on functions $u$ and $\omega$ are ensuring boundedness of a discrete Hardy-type operator from a weighted sequence space $l_{p, u}$ to a weighted sequence space for a wide range of the numerical parameters $p, u$ and $\theta$.

\section{Acknowledgement}

We thank Professor Lars-Erik Persson for several suggestions, which have improved final version of this paper.

\section{References}

[1] Opic B. and Kufner A., "Hardy-Type Inequalities", Pitman Research Notes in Mathematics Series Longman Scientific and Technical, Harlow (1990): 344

[2] Kufner A., Maligranda L. and Persson L.-E., "The Prehistory of the Hardy Inequality", Amer. Math. Monthly. vol. 113(8)(2006): 715-732. 
[3] Kufner A., Persson L-E. and Samko N., "Weighted inequalities of Hardy type, Second Edition" , World Scientific Publishing Co. Pte.Ltd., New Jersey. (2017):459+XX.

[4] Goldman M. L., "Hardy type inequalities on the cone of quasimonotone functions", Khabarovsk Computer Center Fart Eastern Brunch Russian Academy of Science, Research report no 98/31 (1998): 1-69.

[5] Oinarov R., Kalybay A.A., "Three-parameter weighted Hardy type inequalities", Banach Journal Math. vol. 2, no 2 (2008): 85-93.

[6] Gogatishvili A., Mustafayev R., Persson L.-E., "Some new iterated Hardy-type inequalities", J. Func. Spaces Appl. vol. 2012 (2012): 30 .

[7] Gogatishvili A., Mustafayev R., Persson L.-E., "Some new iterated Hardy-type inequalities: the case $\theta=1$ ", J. Inequal. Appl. vol. 2013, no 515 (2013): 29.

[8] Gogatishvili A., Mustafayev R., "Weighted iterated Hardy-type inequalities", Math. Inequal. Appl. vol. 20, no 3 (2017): 683-728.

[9] Prohorov D.V., Stepanov V.D., "Vesovye ocenki klassa sublinejnyh operatorov [Weighted estimates for a class of sublinear operators]", DAN vol. 453, no 5 (2013): 486-488.

[10] Prohorov D.V., Stepanov V.D., "O vesovyh neravenstvah Hardi v smeshannyh normah [On weighted Hardy inequalities in mixed norms]", Tr. MIAN 283(2013): 155-170.

[11] Prohorov D.V., Stepanov V.D., "Ocenki odnogo klassa sublinejnyh integral'nyh operatorov [Estimates for a class of sublinear integral operators]", DAN vol. 456, no 6 (2014): 645-649.

[12] Prohorov D.V., Stepanov V.D., "Vesovye neravenstva dlja kvazilinejnyh integral'nyh operatorov na poluosi i prilozhenija $\mathrm{k}$ prostranstvam Lorenca (Weighted inequalities for quasilinear integral operators on the semi-axis and applications to Lorentz spaces]", Matem.sb. vol. 207, no 8 (2016): 135-162.

[13] Prohorov D. V., "Ob odnom klasse vesovyh neravenstv, soderzhashhih kvazilinejnye operatory [On a class of weighted inequalities containing quasilinear operators]", Tr. MIAN 289(2016): 280-295.

[14] Krepela M., Pick L., "Weighted inequalities for iterated Copson Integral operators [electronic resource]" , (2019). URL: https://www.researchgate.net/publication/332962081 (date of the application: 21.10.2019).

[15] Stepanov V.D., Shambilova G.E., "On weighted iterated Hardy-type operators", Analysis Math. vol. 44, no 2 ( 2018): $273-283$.

[16] Burenkov V.I. and Oinarov R., "Necessary and Sufficient conditions for boundedness of the Hardy-type operator from a weighted Lebesque space to a Morrey-type space", Math. Inequal. Appl. vol. 16, no 1 (2013): 1-19.

[17] Oinarov R. and Kalybay A., "On boundedness of the conjugate multidimensional Hardy operator from a Lebesque space to a local Morrey-type space", Int. J. Math. Anal. vol. 8, no 11 (2014): 539-553.

[18] Bernardis A.L. and Salvador P.O., "Some new iterated Hardy-type inequalities and applications", J. Math. Ineq. vol. 11, no 2 (2017): 577-594.

[19] Canestro M.I.A., Salvador P.O., Torreblanca C.R., "Weighted bilinear Hardy inequalities" , J. Math. Anal. and Appl. 387 (2012): 320-334.

[20] Krepela M., "Iterating bilinear Hardy inequalities", J. Math. Ineq. vol. 60, no 4 (2017): 955-971.

[21] Gogatishvili A., Krepela M., Rastislav O., Pick L., "Weighted inequalities for discrete iterated Hardy operators [electronic resource]" , (2019). URL: https://arxiv.org/abs/1903.04313 (Submitted on 11 March 2019).

[22] Temirhanova A.M., Omarbaeva B.K., "Vesovaja ocenka odnogo klassa kvazilinejnyh diskretnyh operatorov: sluchaj $0<$ $q<\theta<p<\infty, p>1$ [Weighted estimate of a class of quasilinear discrete operators: the case $0<q<\theta<p<\infty$, $p>1$ ]", Vestnik KazNPU im. Abaja vol. 67, no 3 (2019): 109-116. 